\title{
La mesure des arbres et des peuplements forestiers Édition 2021
}

Auteur : RONDEUX Jacques - ISBN : 9782870161708 3ème édition revue et augmentée, 736 pages

Prix : $40 €+$ frais de port.

Presses agronomiques de Gembloux Passage des Déportés, 2, 5030 Gembloux, Belgique. Texte intégral en OPEN ACCESS : https://orbi.uliege.be/handle/2268/262622

Commandes • par le site internet http://pressesuniversitairesdeliege.be/publications/agron omie-gembloux/ • par mail pressesagro.gembloux@uliege.be•par téléphone 00.32.81.62.22.42•chez les libraires

P

rofesseur ordinaire émérite, Jacques Rondeux a enseigné la dendrométrie, la gestion et l'économie forestière à Gembloux Agro-Bio Tech, Université de Liège (Belgique). A consacré près de 40 ans de recherches à la caractérisation et l'évaluation quantitative des peuplements. Auteur de plus de 300 publications et co-auteur de trois ouvrages dédiés aux tables de cubage et de production. A été sollicité au titre de professeur-visiteur dans plusieurs pays africains, ou comme expert. Sa carrière a largement été marquée par le développement de l'inventaire permanent des ressources forestières de Wallonie et du Grand- Duché de Luxembourg. A siégé durant plus de 30 ans au Conseil Supérieur Wallon des Forêts et est correspondant étranger de l'Académie d'Agriculture de France (Section Bois et Forêts). Édité pour la première fois en 1993 « La mesure des arbres et des peuplements forestiers » a fait l'objet d'une révision en 1999. La présente version constitue à la fois une refonte et une importante mise à jour tenant compte de l'évolution des techniques de collecte et d'exploitation des mesures réalisées en forêt et du besoin croissant d'appréhender de nouveaux paramètres, autres que la seule matière ligneuse, dans une perspective d'amélioration de la compréhension de l'écosystème forestier et de la gestion des espaces boisés. L'ouvrage, un des rares en langue française, est davantage conçu comme un manuel de référence en dendrométrie destiné en particulier aux étudiants si souvent aujourd'hui conditionnés à la consultation d'internet et à l'utilisation de logiciels « ready to use ». Il vise aussi un public composé d'enseignants, de chercheurs et de gestionnaires forestiers. Il est délibérément construit sur la base d'une trame abordant les principaux sujets traités d'une manière progressive en ne sacrifiant pas certaines approches qui pourraient être considérées comme appartenant au passé mais pouvant se révéler très utiles pour mieux comprendre la dendrométrie et la faire partager au plus grand nombre. Il est loin le temps où cette science forestière se résumait à la seule " mesure des arbres " ayant pour but précis la connaissance de leur volume. La lecture de l'ouvrage peut se faire à deux vitesses, l'une destinée à un public averti, l'autre privilégiant davantage l'apprentissage.

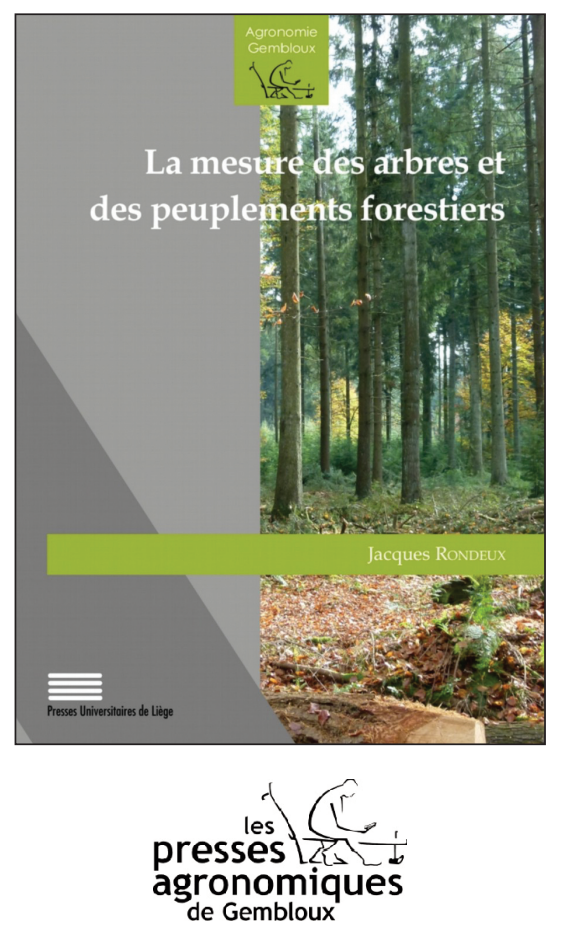

Une édition des Presses universitaires de Liège - Agronomie Gembloux Passage des Déportés, 2 5030 Gembloux (Belgique) $00(32) 81.62 .22 .42$ 